\title{
MOLECULAR-GENETIC ASPECTS OF BREAST CANCER
}

\author{
M. Krasteva, Sv Angelova and Zl Gospodinova \\ Department of Molecular Genetics, Institute of Plant Physiology and Genetics, \\ Bulgarian Academy of Sciences, Sofia, Bulgaria
}

Summary. Breast cancer is the most frequent malignancy among women. Advances in breast cancer knowledge have deciphered the involvement of a number of tumor suppressor genes and proto-oncogenes in disease pathogenesis. These genes are part of the complex biochemical pathways, which enable cell cycle control and maintenance of genome integrity. Their function may be disrupted as a result of alterations in gene sequence or misregulation of gene expression including alterations in DNA methylation pattern. The present review summarizes the main findings on major breast cancer related genes BRCA1/2, p53, ATM, CHEK2, HER2, PIK3CA and their tumorigenic inactivation/activation. The potential clinical importance of these genes with respect to patients' prognosis and therapy are also discussed. The possible implication of other putative breast cancer related genes is also outlined. The first elaborate data on the genetic and epigenetic status of the above mentioned genes concerning Bulgarian patients with the sporadic form of the disease are presented. The studies indicate for a characteristic mutational spectrum in some of the genes for the Bulgarian patients and specific correlation between the status of different genes and clinicopathological characteristics.

Key words: breast cancer, tumor suppressor genes, proto-oncogenes, mutations, DNA hypermethylation, clinical impact

Cancer represents a multistep process that occurs as a result of accumulation of mutations and disruption of the regulatory mechanisms of specific genes involved in normal processes of cell growth and differentiation. The disturbed function of these genes triggers cancer pathogenic mechanisms attributing selective advantages to the transformed cell: resistance to antiproliferative signals, maintenance of proliferative activity in the absence of growth signals, unlimited replicative potential, avoidance of apoptosis, invasion and metastasis, and angiogenesis. The conversion of a normal cell into tumorigenic requires about 6-7 consecutive mutational events. Two major groups of cancer re- 
lated genes have been identified: proto-oncogenes and tumor suppressor genes, which are functionally interconnected in a complex regulatory network of biochemical interactions. Proto-oncogenes are positive regulators of the cell cycle and tumorigenic alterations enhance their expression. Contrariwise, tumor suppressors inhibit cells growth and proliferation and their loss-of-function may lead to transformation.

Advances in modern breast cancer knowledge have revealed the implication of several tumor suppressor genes and proto-oncogenes in disease pathology. They are an integral part of the complex biochemical pathways enabling cell cycle control and maintenance of genome integrity.

Most of the breast cancer cases are sporadic, due to somatic mutations in a large number of genes. The sporadic disease has a later onset, the tumors are mostly unilateral and unifocal and no other family members are usually affected. Familial cases are due to inherited (germ-line) mutations. The major familial breast cancer related genes are $B R C A 1$ and $B R C A 2$. Inherited carcinomas have an early onset; they are more aggressive, mostly bilateral and multi-focal. Such tumors are often accompanied with carcinomas of other sites; pedigree analysis reveals affected family members. A small part of breast cancers are monogenic, a part of inherited syndromes, such as Cowden syndrome (due to a germ-line mutation in PTEN), Reifenstein syndrome (in MLH1), Li-Fraumeni syndrome (in p53), Ataxia-telangiectasia (in ATM), на Peutz-Jeghers syndrome (in STK11).

\section{BRCA1 AND BRCA2 TUMOR SUPPRESSOR GENES}

The breast cancer susceptibility genes BRCA1 and BRCA2 have been discovered in the middle of the 90 s of the last century and have been mapped to chromosomes 17 (17q21) and 13 (13q12-13), respectively. BRCA1 encompasses about 100 $\mathrm{kb}$ of genome DNA and is organized into 22 coding and 2 non-coding exons. The encoded protein is composed of 1863 amino acids. BRCA2 consists of 27 exons and codes for 3418 amino acids protein. Both genes belong to the class of tumor suppressors named "guardians of the genome". BRCA1/2 proteins have specific DNA-binding domains and are involved in transcription control, DNA repair, apoptosis and others [14]. BRCA1 has more than 15 target genes among which are BRCA2, p53 and ATM, thus being nominated as a key regulator in maintenance of cell balance [32].

When inactivated, $B R C A 1$ and $B R C A 2$ are tumorigenic. Currently several thousands of $B R C A 1 / 2$ mutations have been discovered (Breast Cancer Information Core Database). Breast cancer related BRCA1/2 mutations are mainly gem-line. They spread throughout the whole genes and no "hot spots" could be differentiated. Most BRCA1 mutations lead to the synthesis of abnormally short non-functional variants (frameshift mutations, small deletions and insertions) or normal in size but functionally inactive proteins (missense mutations). BRCA1 mutational frequency may vary depending on the family burden and the age of affected persons. The carriers of BRCA1 mutations have an early breast cancer onset, clinical characteristics are indicative of an aggressive disease, often both breasts are affected [53]. BRCA1- 
positive tumors are mainly ductal, with a high grade of malignancy, negative ER and PR receptor status, HER2 overexpression and often carry a $p 53$ mutation [21]. Single studies have found some specific characteristics of BRCA2-positive tumors, related to gene expression and immunohistochemical profile, distinguishing them from other breast carcinomas [21].

The overall lifetime risk of developing breast cancer for the carriers of $B R C A 1$ mutation is $46 \%$, and for BRCA2 - 43\% [17]. BRCA1/2 mutation carriers have also an increased risk of developing other carcinomas including ovarian, pancreatic, colorectal and others [13].

It is surprising that in contrast to familial breast cancer, no or limited somatic $B R C A 1$ mutations have been found in the sporadic form of the disease. Nevertheless, $B R C A 1 \mathrm{mRNA}$ level has been found to be reduced or absent in invasive sporadic breast tumors, thus assigning a role of BRCA1 in sporadic breast cancer as well. This observation is suggestive that other, alternative loss-of-function mechanisms could be involved, including DNA hypermethylation, dysregulation of transcriptional activators/repressors binding to the BRCA1 locus or post-transcriptional processes. Hypermethylation in BRCA1 promoter in sporadic breast cancer is firstly described by Dobrovic et Simpfendorfer [20]. This epigenetic abnormality is related to inhibition of gene expression and decreased $B R C A 1 \mathrm{mRNA}$ level [43]. The reported frequency of $B R C A 1$ promoter hypermethylation in sporadic breast carcinomas is in the range of 9 to $59 \%$. Though tumors with BRCA1 promoter hypermethylation display various cancer phenotypes, several most frequent features could be outlined. Thus, though not a rule, most studies demonstrated that $B R C A 1$ hypermethylation correlated with lack of estrogen and progesterone receptor expression and is most frequently present in younger women, below the age of 50 . As this in some way resembles the familial $B R C A 1$ mutated tumors, it has been suggested that $B R C A 1$ hypermethylated carcinomas might phenocopy familial [55].

Bozhanov et al. [12] studied BRCA1 mutational status in a large group of Bulgarian patients with sporadic breast cancer and found one single $A>T$ polymorphism mapped in intron 4 in position 19,479. The first data on BRCA1 methylation status in Bulgarian patients with sporadic breast cancer was provided by Krasteva et al. [26]. The frequency, the authors found, was a little below the average BRCA1 hypermethylation frequency $-17.04 \%(23 / 135)$. Patients with hypermethylation in $B R C A 1$ displayed favorable clinical status as their tumors were smaller in size $(P=0.066)$, lacked $p 53$ gene mutations $(P=0.073)$ and were of lobulartype $(P=0.046)$. The presence of hypermethylation was weakly associated with better overall survival $(P=0.2)$.

\section{P53 TUMOR SUPPRESSOR GENE}

The $p 53$ gene has been identified more than 30 years ago and is the first tumor suppressor found mutated in human carcinomas. The human $p 53$ is single-copy spreading over $20 \mathrm{~kb}$ of DNA. It is localized in the short arm of chromosome 17 , in 
position $17 \mathrm{p} 13.1$ and is composed of 11 exons varying in size from 22 to $1278 \mathrm{bp}$. The encoded phosphoprotein is relatively small composed of 393 amino acids. The functional molecule is a tetramer organized into several specific functional domains, typical for the transcription factors.

Under normal conditions, p53 is inactive and unstable. Its activation requires conformational changes and could be induced by genotoxic agents or other forms of genotoxic stress like hypoxia, nucleotide depletion, oncogenic activation, microtubules disruption, loss of normal cellular contacts, cell aging and others [28]. It has been shown that the pathways engaged in the processes of p53 activation include sensors of DNA double strand breaks such as ATM (or ATR) and CHEK2. When activated p53 could be employed in protein complexes with other related proteins such as BRCA1. The p53 target DNA sequence is relatively variable and was found in several hundreds of genes whose function has been differentially modulated (induced or repressed) depending on the cellular type, the nature of the stress, and the level of DNA damage [28].

The gene has a central role in maintaining of genome integrity and acts as a "guardian of the genome". In response to cellular stress, depending on the level of damage, p53 determines the fate of cells either towards survival, involving cell-cycle arrest and DNA damage repair, or cell suicide, carried out by apoptosis. Roles in DNA repair [48], angiogenesis [31], cell differentiation and morphogenesis [5] are also attributed to $\mathrm{p} 53$.

In cancer cells the normal p53 function could be disrupted by various mechanisms: somatic or germ-line mutations (single base changes, small or large deletions, structural rearrangements and insertions); formation of stable protein complexes between the wild type p53 and viral oncoproteins (such as T antigen, E1B, E6, HBxAg, EBNA-5, BZLF1); localization of wild type p53 in cell cytoplasm, alterations in p53 regulators and targets, aberrant promoter hypermethylation and others.

The loss of normal p53 function disrupts altogether the control over the cell cycle. No monitoring over the structural rearrangements in DNA is carried out. The cells pass unimpeded the cellular checkpoints. Genome instability arises; mutations in different oncogenes and tumor suppressor genes accumulate, releasing the processes of tumorigenesis. The disturbance of normal p53 activities leads to selective advantage of cells depriving them of the normal mechanisms of cellular control.

Somatic p53 mutations are the most frequent tumorigenic event. They are found in tumors of the colon, stomach, liver, lung, breast, brain and others with a frequency ranging from $5.6 \%$ to $48.3 \%$ [39]. The most common mutations in tumor suppressor genes are nonsense mutations, deletions and insertions, i.e. loss-offunction mutations that produce either an absent or a truncated protein product. With respect to this, $p 53$ has an unusual mutation spectrum since more than $70 \%$ of the p53 mutations reported so far are single base missense substitutions leading to the synthesis of altered proteins. More than $90 \%$ of the mutations are clustered within the conserved regions of exons $5 \pm 8$, affecting amino acids in or near the central 
DNA-binding domain. Among these regions several mutation "hot spots" have been identified.

Mutations in $p 53$ could be inherited. The inheritance of a mutant $p 53$ allele increases cancer risk. Tumors associated with inherited p53 mutations are those of the breast, soft tissues, brain, bones and other. Germ-line p53 mutations are specifically associated with Li-Fraumeni and Li-Fraumeni-like syndromes [34]. LiFraumeni is a rare, autosomal dominant disorder characterized with the formation of diverse multiple mesenchymal and epithelial neoplasms in family members at an early age, before 45 . Germ-line $p 53$ mutations have been identified in about $70 \%$ of Li-Fraumeni and 25\% Li-Fraumeni-like families [39]. By the use of molecular genetic screening techniques in members of Li-Fraumeni families that exhibit high incidence of cancer, the risk of cancer developing and passing the mutant $p 53$ allele to the offspring could be determined. Such cancer-prone, though asymptomatic, individuals need a special prophylaxis control.

Though the existing literature discrepancies, the p53 status overall has been proposed as an independent marker for poor prognosis. Meta-analysis of over 3500 patients with breast cancer revealed that $p 53$ mutations correlate with lower survival and this effect was independent of other risk factors [42]. Carcinomas with p53 mutations are associated with more aggressive tumor phenotype, early metastasizing and more frequent recurrents.

In the first study on $p 53$ mutational status in Bulgarian patients with sporadic breast cancer, the authors found $22.07 \%$ frequency of mutations in a group of 145 patients [12]. The mutational spectrum of $p 53$ was rather heterogeneous as missense and nonsense mutations, deletion and insertion frameshifts, a splice-site mutation, silent substitutions, exon and intron polymorphisms were found. Five of the p53 mutations were novel and had never been reported in any type of human cancer. That presumes specific spectrum of $p 53$ mutations for the Bulgarian patients. Comparison with clinical parameters showed that mutated p53 predicts an aggressive clinical course of breast carcinomas. In support to this was the observation that $p 53$ positive women had larger $(P=0.033)$ and poorly differentiated tumors $(P=0.001)$. Mutated $p 53$ decreased overall survival of breast cancer patients $(P=$ 0.048 ), though multivariate analysis failed to show an independent effect. This correlation was even stronger in the subgroup above 50 years $(P=0.017)$ and in the women with $\mathrm{T} 1$ tumors $(P=0.014)$.

In addition to genetic status, Krasteva et al. [27] studied the epigenetic promoter methylation status of $p 53$ gene in Bulgarian patients with sporadic breast cancer. No aberrant hypermethylation was found. Authors concluded that with respect to $p 53$, the impact of genetic mechanisms in sporadic breast carcinogenesis seems predominating. However, other epigenetic mechanisms may affect the functional activity of $p 53$ in the process of tumorigenesis, such as histone modifications, alterations in chromatin condensation, RNA interference and others. 


\section{ATM TUMOR SUPPRESSOR GENE}

ATM gene is localized to the long arm of chromosome 11 at a position 11q22q23. It is composed of 66 exons (62 of which are coding) and covers approximately $150 \mathrm{~kb}$ of genomic DNA. ATM is one of the largest human genes but occupies a relatively compact genome region. The protein is a $350 \mathrm{kDa}$ phosphoprotein - serine/ threonine kinase composed of 3056 amino acids and belongs to the phosphoinositide 3-kinase superfamily (PI3-kinases). Structural and functional features of ATM protein include presence of ATP- and DNA binding sites, two substrate-binding regions and three differentiated regions (domains) - FAT, FATC, PI3K. ATM kinase is found in cells nuclei, near to DNA, and outside the nucleus - in cytoplasm, attached to different cellular structures or associated with $\beta$-adaptin.

ATM is a sensor of double-strand breaks in DNA. In response to them, it activates and triggers cellular signaling pathways, thus providing active control at all cell cycle checkpoints, repair and apoptosis. Once activated, ATM in turn activates its target genes by phosphorylation [29, 30]. ATM target genes are a number of sensory genes, mediators, transducers and key effectors, among which $p 53, B R C A 1$, BRCA2, CHEK2 [23].

The carrying of homozygous ATM mutations is responsible for the autosomal recessive disease Ataxia Telangiectasia $(\mathrm{A}-\mathrm{T})$. The clinical features are complex including brain damages, telangiectasias and recurrent respiratory and middle ear infections, as a result of severe disorders of the immune status. Affected individuals are prone to the development of T-cell-prolymphocytic leukemia, B-cell chronic lymphocytic leukemia and also sporadic colon cancer with microsatellite instability. Heterozygous carriers show moderate radiosensitivity and increased predisposition to cancer.

At present, over 3000 alterations in ATM gene are described (UniProtKB, Ensembl, NGMD databases). Dominating are these, established in gene non-coding sequences - over 2200. ATM mutations associated with breast cancer are primarily germ-line. Although more rarely, somatic mutations are also described, but with considerably lower frequency. Mutations occur along the entire length of the gene, without established "hot spots". The prevailing ATM mutations, related to breast cancer, are of two types - truncating and missense. Truncating mutations are more typical for patients with A-T [11].

Studies regarding ATM mutations in breast cancer sporadic cases are limited. Data found are contradictory and indicate base substitutions of the missense type and intron polymorphisms with a relatively low frequency [9].

Currently, the data on the epigenetic attribution in ATM loss-of-function in breast tumors are incomplete and inconsistent. It is found that promoter hypermethylation is the reason for ATM lack of expression in head and neck tumors [2]. Data showing suppressed expression of ATM in breast tumors are also published [6].

It is considered that ATM could potentially be an informative indicator for the effect of radiation therapy. Because of the fact that ATM is a sensor of double-strand 
breaks, which are caused mainly by ionizing radiation, when functioning normally in tumor cells good therapeutic response could be expected. Contrariwise, for carriers of ATM mutations increased risk of radiation-induced late effects exists [22].

According to data of Bozhanov et al. [12], 11 mutations in ATM gene $(7.6 \%$, $11 / 145$ ) were found in a group of Bulgarian patients with breast cancer. The detected frequency was similar to the frequency in other studied populations. ATM mutational spectrum in Bulgarian patients with breast cancer was homogeneous. The registered alterations are only in exons 15 and 39, and all of them are base substitutions, leading to amino acid replacement (missense): two Ser707Pro, five Asp1853Asn and four Asp1853Asn/ivs38-8T >C. Patients with mutant ATM have predominantly low grade $(P=0.032)$ and lobular $(P=0.09)$ tumors, which are considered to be favorable clinical characteristics. Mutant ATM status prevails in PIK3CA-positive patients $(P=0.098)$. Decrease in overall survival of patients with ATM mutation was not detected, excepting the subgroup with negative ER $(P=0.021)$. In a single study on epigenetic promoter methylation status of ATM gene in Bulgarian patients with breast cancer, such was not registered [27].

\section{CHEK2 TUMOR SUPPRESSOR GENE}

The tumor suppressor gene CHEK2 maps to the long arm of chromosome 22 at position 22q12.1 and consists of 14 coding exons. It encodes a protein kinase, comprising 543 amino acids with three individual conservative domains: an N-terminal regulatory domain, rich of serine, threonine and glutamine (SCD); FHA-domain, responsible for protein-protein interactions and large C-terminal kinase domain. CHEK2 is a nuclear protein, which is expressed through all stages of the cell cycle. The protein is present in lower levels in non-dividing cells [33].

CHEK2 plays a significant role in the regulation of the cell response in the presence of DNA double strand breaks. It carries out the control at all major checkpoints of the cell cycle, regulates the repair process and directs the cell to apoptosis [40]. ATM is a main activator of CHEK2 and its activation is accomplished by phosphorylation of Thr68 in the SCD domain $[1,35]$. Under normal conditions CHEK2 exists as an inactive dimer. As a result of phosphorylation and subsequent transphosphorylation in the FHA domain, release of active CHEK2 monomers occurs [58]. Once activated CHEK2 transmits the signal for DNA damage to the effectors resulting in cell cycle arrest in G1/S, S and G2/M, followed by repair process or apoptosis. The most important and best studied substrates of CHEK2 are: p53, BRCA1 and CDC25.

In 1999 Bell et al. [8] found germ-line mutations (1100delC and I157T) in CHEK2 gene in patients with Li Fraumeni syndrome and negative $p 53$ status. Based on this observation an association between the detected mutations in CHEK2 and this hereditary syndrome was initially assumed. However, later it became clear that CHEK2 mutations are also present in patients with familial and sporadic breast cancer $[4,52]$. 
According to the Ensemble database a total of 1924 alterations in human CHEK2 gene are so far registered. Most of them are localized in noncoding sequences while others are scattered in the coding gene regions without formation of "hot spots". It is established that among the mutations found in CHEK2 in patients with breast cancer deletions, substitutions or insertion of single nucleotides are dominating. Most of the mutations are germ-line, but also somatic mutations are found, though with considerably lower frequency. Some mutational variants, moderately associated with an increased risk of breast cancer development are found and characterized: 1100delC, I157T, S428F, IVS2+1G>A and del5395 [10, 18, 25, 36, 49, 56]. Interesting is the fact that these mutations show certain population specificity and are not found or are found in a very low frequency in patients with breast cancer from certain populations. Mutations in CHEK2 are also detected in patients with osteosarcoma, lung, ovarian cancer and prostate cancer.

CHEK2 promoter hypermethylation is found in tumors of the lungs, vulva and Hodgkin lymphoma. In limited number of publications promoter hypermethylation is considered as probable epigenetic mechanism for loss of function of CHEK2 in breast tumors.

At present, the significance of the mutant CHEK2 status for the clinical manifestation and survival of breast cancer patients is not elucidated. Available data encompass only well-known mutant variants and often reliable assessment is lacking because of the found too low frequency.

The first data on CHEK2 mutational spectrum in Bulgarian patients with breast cancer are provided by Angelova et al. [7]. The mutational spectrum found in CHEK2 shows well-defined specificity to Bulgarian patients. The most frequent and wellcharacterized mutant variants, associated with CHEK2, are absent in Bulgarian patients. The authors found three novel mutations in CHEK2 gene: Glu79Glu $(A \rightarrow G)$, Thr476Met $(\mathrm{C} \rightarrow \mathrm{T})$, Ala507Gly $(\mathrm{C} \rightarrow \mathrm{G})$. It was shown that $\mathrm{CHEK2}$ status significantly correlates with some of the clinicopathological parameters, including lobular histological type $(P=0.04)$ and positive $P R$ status $(P=0.092)$, as well as with ATM mutational status $(P=0.046)$. Multivariate survival analysis indicated independent 3.295 fold increased risk of death from breast cancer in patients with CHEK2 alterations $(95 \% \mathrm{Cl} 0.850-12.778, \mathrm{p}=0.085)$. Authors did not detect aberrant methylation in CHEK2 promoter in studied group of Bulgarian patients.

\section{HER2 PROTO-ONCOGENE}

The proto-oncogene HER2, known also as erbB-2, is localized on the long arm of chromosome 17, in position 17q11.2-17q12.0. Under normal conditions the two copies of the gene produce low amounts of membrane tyrosine kinase receptor protein. When activated, the HER2 receptor forms heterodimers with other members of the HER family, thus triggering a cascade of events, which transmit the growth signal to the nucleus of the cell through the process of signal transduction. HER2 is 
involved in cell control, modulation of cell polarity, intracellular interactions, proliferation and morphogenesis [37, 38].

Overexpression of HER2 over the membrane leads to self-association of the receptor in a concentration-dependent manner. In such a state, the receptor is constitutively activated, independent of the ligand. The increased level of HER2 receptor results in serious disruption in cell proliferation and differentiation: increase in proliferative activity; angiogenesis; alterations in intracellular interactions; increase cell migration ability and metastasis; sustainability to apoptosis; malignant cell transformation. HER2 activation might be due to gene amplification, changes in gene structure or in transcriptional activity. HER2 amplification is the major mechanism for its activation and has been observed in $92 \%$ of studied breast cancers that were shown to overexpress HER2 protein [3].

Analysis of HER2 status in human tumors showed that approximately 10 to $30 \%$ of the studied carcinomas of the breast, lung, kidneys, prostate, salivary gland and others, carry amplified/overexpressed HER2. In breast cancer, HER2 gene amplification and protein overexpression occur in approximately $25-30 \%$ of the cases and is generally associated with poor prognosis [51]. HER2 overexpression was more frequent in younger women, under the age of 35 [24]. Amplification/overexpression of HER2 is shown to correlate with several negative prognostic variables [44] and is associated with shorter survival, higher recurrence rates and lower response to chemotherapy and hormone therapy [16]. From a clinical point of view, HER2 has become important mainly as a target for antibody-based therapy with trastuzumab. Adjuvant trastuzumab treatment of primary HER2 positive patients with breast cancer has been shown to markedly improve outcome [54]. At present, determination of HER2 status in every breast cancer patient to select for adjuvant treatment with trastuzumab is becoming a standard in BC clinical practice worldwide.

The study of Bozhanov et al. [12] included HER2 status as well. The authors revealed that $21.21 \%$ of tumor samples overexpress the proto-oncogene. They found that HER2 overexpression is related to an aggressive clinical course of breast carcinomas. Consistent with previous data, HER2 positive tumors were significantly larger than HER2 negative $(P=0.0004)$. However, conversely, they were mainly ER positive $(P=0.011)$. Though related to aggressive breast carcinogenesis, HER2 positive status did not correlate significantly with survival. In contrast to previously reported data [45], they found that HER2 overexpression occurred in older patients, over or at the age of $50(P=0.009)$.

\section{PIK3CA PROTO-ONCOGENE}

It has been recently established that the proto-oncogene PIK3CA is mutated in more than $30 \%$ of breast carcinomas, which makes it the most highly mutable gene in breast cancer [15]. PIK3CA is a lipid kinase, whose oncogenic activity is due mainly to missense mutations in specific "hot spots" mainly in exons 9 and 20 . The 
mutant PIK3CA interacts and activates the AKT kinase, thus leading to stimulation of cell proliferation, inhibition of apoptosis and initiation of tumorigenesis [47]. The so far established mutations in PIK3CA proto-oncogene are somatic, presented only in the tumor tissue. Some studies found a correlation between PIK3CA mutant status and other cancer related genes. Thus it has been shown that mutations in PIK3CA and $p 53$ are incompatible and mutually exclusive [50], whereas HER2 overexpression is often accompanying PIK3CA mutations, presuming their interdependence in breast carcinogenesis [46].

The first data on mutational PIK3CA status in Bulgarian patients with breast cancer were obtained after direct sequencing of mutation "hot spots" in exons 9 and 20 [12]. The determined frequency of PIK3CA mutations was 31\% (45/145). The mutations were of the type E542K, E545K and H1047R. The authors found that the mutant PIK3CA significantly correlates with positive $P R$ status $(P=0.047)$.

\section{OTHER POTENTIAL BREAST CANCER RELATED GENES}

Although the initial enthusiasm followed the discovery of BRCA genes and their relation to the inherited breast/ovarian cancer, many affected families do not carry pathogenic $B R C A$ mutations. It seems that alterations in BRCA1/2 genes are responsible for about $15 \%$ of breast cancer cases having first-grade relative who has developed the disease [41]. This is presumable for the implication of other genes in familial breast cancer disease etiology. Such potential candidates are BRIP1, $P A L B 2$ and $R A D 51 C$ genes, because of their functional interconnection to BRCA1 and $B R C A 2$, involvement in DNA repair processes and relation to breast cancer risk [57]. However, at this stage, the data in this respect are still limited.

In the genetic predisposition to breast cancer, potential significance have also some other tumor suppressor genes, among which Rb, p16, PTEN, STK11, WT-1, $A P C$, IGFR2 and others. In addition to this, several classes of low penetrating genes, which can be potentially associated with breast cancer, have been identified [19]. These are genes, engaged in metabolic cellular pathways (cytochrome p450, GST and NAT); genes, involved in the pathways of estrogen, progesterone and androgen receptors; genes with a role in immunomodulatory mechanisms (HLA, TNFa, HSP70); genes, participating in iron metabolism (HFE and TFR), vitamin D receptor gene (VDR) and others. It is anticipated that other genes may be involved, to a less or greater extent, in disease etiology. Such genes are about to be identified and studied. 


\section{REFERENCES}

1. A h n, J. Y. et al. Threonine 68 phosphorylation by ataxia telangiectasia mutated is required for efficient activation of Chk2 in response to ionizing radiation. - Cancer Res., 60, 2000, № 21, 59345936.

2. A i, L. et al. Ataxia-telangiectasia-mutated (ATM) gene in head and neck squamous cell carcinoma: promoter hypermethylation with clinical correlation in 100 cases. - Cancer Epidemiol. Biomarkers Prev., 13, 2004, № 1, 150-156.

3. A I b a i n, K. S. et al. HER2 overexpression in Breast Cancer. - Genetech Inc., 1998.

4. All in e $n, M$. et al. Mutation analysis of the CHK2 gene in families with hereditary breast cancer. Br. J. Cancer, 85, 2001, № 2, 209-212.

5. A I m o n d, N. et V. Rotter. Involvement of p53 in cell differentiation and development. - Biochim. Biophys. Acta /Reviews on Cancer, 1333, 1997, № 1, 1-27.

6. An g e le, S. et al. Abnormal expression of the ATM and TP53 genes in sporadic breast carcinomas. - Clin. Cancer Res., 6, 2000, 3536-3544.

7. A n g e I o va, S. G. et al. CHEK2 gene alterations independently increase the risk of death from breast cancer in Bulgarian patients. - Neoplasma, 59, 2012, № 6, 622-630.

8. B e I I, D. W. et al. Heterozygous germ line hCHK2 mutations in Li-Fraumeni syndrome. Science, 286, 1999, № 5449, 2528-2531.

9. $B$ e r $n$ s t e i n, J. L. et al. ATM variants $7271 T>G$ and IVS10-6T $>G$ among women with unilateral and bilateral breast cancer. - Br. J. Cancer, 89, 2003, 1513-1516.

10. B o g d a n o v a, N. et al. Association of two mutations in the CHEK2 gene with breast cancer. - Int. J. Cancer, 116, 2005, № 2, 263-266.

11. $B ø r r$ e s e $n, A$. L. et al. Breast cancer and other cancers in Norwegian families with ataxia-telangiectasia. - Genes Chromosomes Cancer, 2, 1990, № 4, 339-340.

12. B o z h a $\mathrm{n}$ o v, S. / Angelova S. et al. Alterations in p53, BRCA1, ATM, PIK3CA, and HER2 genes and their effect in modifying clinicopathological characteristics and overall survival of Bulgarian patients with breast cancer. -J. Cancer Res. Clin. Oncol., 136, 2010, 1657-1669.

13. B r o s e, M. S. et al. Cancer Risk Estimates for BRCA1 Mutation Carriers Identified in a Risk Evaluation Program. - J. Natl. Cancer Inst., 94, 2002, 1365-1372.

14. $\mathrm{C} \mathrm{a} \mathrm{b} \mathrm{I} \mathrm{e,} \mathrm{P.} \mathrm{L.} \mathrm{et} \mathrm{al.} \mathrm{Novel} \mathrm{consensus} \mathrm{DNA-binding} \mathrm{sequence} \mathrm{for} \mathrm{BRCA1} \mathrm{protein} \mathrm{complexes.} \mathrm{-} \mathrm{Mol.}$ Carcinog., 38, 2003, № 2, 85-96.

15. C a m p b e II, I. G. et al. Mutation of the PIK3CA gene in ovarian and breast cancer. - Cancer Res., 64, 2004, 7678-7681.

16. C a r r, J. A. et al. The association of HER-2/neu ampliffication with breast cancer recurrence. -Arch. Surg., 135, 2000, 1469-1474.

17. $\mathrm{C}$ h e n, S. et al. Characterization of BRCA1 and BRCA2 mutations in a large United States sample. - J. Clin. Oncol., 24, 2006, № 6, 863-871.

18. $\mathrm{C} \mathrm{y} \mathrm{b} \mathrm{u} \mathrm{I} \mathrm{s} \mathrm{k} \mathrm{I,} \mathrm{C.} \mathrm{et} \mathrm{al.} \mathrm{A} \mathrm{deletion} \mathrm{in} \mathrm{CHEK2} \mathrm{of} 5,395$ bp predisposes to breast cancer in Poland. Breast Cancer Res. Treat., 102, 2007, № 1, 119-122.

19. $\mathrm{d}$ e J o $\mathrm{n} \mathrm{g}, \mathrm{M}$. M. et al. Genes other than BRCA1 and BRCA2 involved in breast cancer susceptibility. - J. Med. Genet., 39, 2002, 225-242.

20. D o b r o v I c, A. et D. Simpfendorfer. Methylation of the BRCA1 Gene in Sporadic Breast Cancer. - Cancer Res., 57, 1997, 3347-3350.

21. $\mathrm{H}$ o $\mathrm{n} \mathrm{r}$ a d o, E. et al. Pathology and gene expression of hereditary breast tumors associated with BRCA1, BRCA2 and CHEK2 gene mutations. - Oncogene, 25, 2006, № 43, 5837-5845.

22. I a $\mathrm{n} \mathrm{u} z \mathrm{zi}, \mathrm{C}$. M. et al. ATM mutations in female breast cancer patients predict for an increase in radiation-induced late effects. - Int. J. Radiat. Oncol. Biol. Phys., 52, 2002, № 3, 606-613. 
23. K a s t a n, M. B. et J. Bartek. Cell-cycle checkpoints and cancer. - Nature, 432, 2004, № 7015, 316-323.

24. K i I i n c, N. et M. Yaldiz. P53, c-erbB-2 expression and steroid hormone receptors in breast carcinoma: correlations with histopathological parameters. - Eur. J. Gynaecol. Oncol., 25, 2004, 5, 606-610.

25. K i I p i v a a r a, O. et al. CHEK2 variant I157T may be associated with increased breast cancer risk. - Int. J. Cancer, 111, 2004, 543-547.

26. $\mathrm{K}$ r a s t e $\mathrm{v}$ a, M. E. et al. Breast cancer patients with hypermethylation in the promoter of BRCA1 gene exhibit favorable clinical status. - Neoplasma, 59, 2012, № 1, 85-91.

27. $\mathrm{K}$ r a s t e $\mathrm{v}$ a, M. et al. Aberrant promoter methylation in $\mathrm{p} 53$ and ATM genes was not associated with sporadic breast carcinogenesis in Bulgarian patients. - J. BioSci. Biotech., 3, 2014, № 2, 105-109.

28. L a c r o i x, M., R. A. Toillon et G. Leclercq. P53 and breast cancer, an update. - Endocr. Relat. Cancer, 13, 2006, № 2, 293-325.

29. $\mathrm{L}$ a v i n, M. F. et S. Kozlov. ATM activation and DNA damage response. - Cell Cycle, 6, 2007, № 8, 931-942.

30. $\mathrm{L}$ a $v$ i $\mathrm{n}, \mathrm{M}$. F. The Mre11 complex and ATM: a two-way functional interaction in recognising and signaling DNA double strand breaks. - DNA Repair (Amst), 3, 2004, № 11, 1515-1520.

31. $L$ a w I e r, J. et al. Thrombospondin-1 gene expression affects survival and tumor spectrum of $p 53-$ deficient mice. - Am. J. Pathol., 159, 2001, 1949-1956.

32. L i, S. et al. Functional link of BRCA1 and ataxia telangiectasia gene product in DNA damage response. - Nature, 406, 2000, № 6792, 210-215.

33. L u k a s, C. et al. DNA damage-activated kinase Chk2 is independent of proliferation or differentiation yet correlates with tissue biology. - Cancer Res., 61, 2001, № 13, 4990-4993.

34. M a I k i n, D. et al. Germ line p53 mutations in a familial syndrome of breast cancer, sarcomas, and other neoplasms. - Science, 250, 1990, № 4985, 1233-1238.

35. $\mathrm{M}$ a t s u o k a, S. et al. Ataxia telangiectasia-mutated phosphorylates Chk2 in vivo and in vitro. Proc. Natl. Acad. Sci. U S A, 97, 2000, № 19, 10389-10394.

36. $\mathrm{M}$ e i j e r s-H e I j b o e r, $\mathrm{H}$. et al. Low-penetrance susceptibility to breast cancer due to CHEK2(*)1100delC in noncarriers of BRCA1 or BRCA2 mutations. - Nat. Genet., 31, 2002, № 1, 55-59.

37. $\mathrm{M} \mathrm{u}$ t h u s w a $\mathrm{m}$ y, S. K. et al. ErbB2, but not ErbB1, reinitiates proliferation and induces luminal repopulation in epithelial acini. - Nat. Cell Biol., 3, 2001, 785-792.

38. O f ft e r d in g e r, M., S. M. Schneider et T. W. Grunt. Heregulin and retinoids synergistically induce branching morphogenesis of breast cancer cells cultivated in 3D collagen gels. - J. Cell Physiol., 195, 2003, 260-275.

39. O I i v i e r, M. et al. The IARC TP53 database: new online mutation analysis and recommendations to users. - Hum. Mutat., 19, 2002, 607-614.

40. O' D r I s c o II M. et P. A. Jeggo. The role of double-strand break repair - insights from human genetics. - Nat. Rev. Genet., 7, 2006, № 1, 45-54.

41. P e t $\mathrm{o}$, J. et al. Prevalence of BRCA1 and BRCA2 gene mutations in patients with early-onset breast cancer. - J. Natl. Cancer Inst., 91, 1999, № 11, 943-949.

42. P h a r o a h, P. D., N. E. Day et C. Caldas. Somatic mutations in the p53 gene and prognosis in breast cancer: a meta-analysis. - Br. J. Cancer, 80, 1999, 1968-1973.

43. R i c e, J. C., K. S. Massey-Brown et B. W. Futscher. Aberrant methylation of the BRCA1 CpG island promoter is associated with decreased BRCA1 mRNA in sporadic breast cancer cells. - Oncogene, 17, 1998, 1807-1812.

44. R o s s, J. S. et al. The Her-2/neu gene and protein in breast cancer: biomarker and target of therapy. - Oncologist, 8, 2003, 307-325. 
45. R u d o I p h, P. et al. Correlation between p53, c-erbB-2, and topoisomerase II alpha expression, DNA ploidy, hormonal receptor status and proliferation in 356 node-negative breast carcinomas: prognostic implications. - J. Pathol., 187, 1999, 207-216.

46. S a a I, L. H. et al. PIK3CA mutations correlate with hormone receptors, node metastasis, and ERBB2, and are mutually exclusive with PTEN loss in human breast carcinoma. - Cancer Res., 65, 2005, 2554-2559.

47. S a n s a I, I. et W. R. Sellers. The biology and clinical relevance of the PTEN tumor suppressor pathway. - J. Clin. Oncol., 22, 2004, 2954-2963.

48. S e o, Y. R. et H. J. Jung. The potential roles of p53 tumor suppressor in nucleotide excision repair (NER) and base excision repair (BER). - Exp. Mol. Med., 36, 2004, № 60, 505-509.

49. $\mathrm{S} \mathrm{h}$ a a $\mathrm{g}$, A. et al. Functional and genomic approaches reveal an ancient CHEK2 allele associated with breast cancer in the Ashkenazi Jewish population. - Hum. Mol. Genet., 14, 2005, № 4, 555563.

50. S i n g h, B. et al. p53 regulates cell survival by inhibiting PIK3CA in squamous cell carcinomas. Genes Dev., 16, 2002, 984-993.

51. S I a m o n, D. J. et al. Human breast cancer: correlation of relapse and survival with amplification of the HER-2/neu oncogene. - Science, 235, 1987, 177-182.

52. S o d h a, N. et al. Screening hCHK2 for mutations. - Science, 289, 2000, № 5478, 359.

53. S t o p p a - L y o $n \mathrm{n}$ e t, D. et al. Familial invasive breast cancers: worse outcome related to BRCA1 mutations. - J. Clin. Oncol., 18, 2000, 4053-4059.

54. T u m a, R. S. Trastuzumab trials steal show at ASCO meeting. - J. Natl. Cancer Inst., 97, 2005, 870-871.

55. T u r n e r, N., A. Tutt et A. Ashworth. Hallmarks of ,BRCAness' in sporadic cancers. - Nature Reviews of Cancer, 4, 2004, 814-819.

56. W e i s c h e r, M. et al. Increased risk of breast cancer associated with CHEK2*1100delC. $-\mathrm{J}$. Clin. Oncol., 25, 2007, № 1, 57-63.

57. W o $\mathrm{ng}, \mathrm{M}$. W. et al. BRIP1, PALB2, and RAD51C mutation analysis reveals their relative importance as genetic susceptibility factors for breast cancer. - Breast Cancer Res. Treat., 127, 2011, № 3, 853-859.

58. X u, X., L. M. Tsvetkov et D. F. Stern. Chk2 activation and phosphorylation-dependent oligomerization. - Mol. Cell Biol., 22, 2002, № 12, 4419-4432.

\section{Corresponding author:}

Maria Emileva Krasteva

Acad. G. Bonchev Str.

BG - 1113 Sofia

(+3592) 9746229/238

助 $(+3592) 9785516$

e-mail: maria_krasteva@abv.bg 